\title{
Screening for down syndrome using trophoblast retrieval and isolation of the cervix: preliminary study
}

\author{
Min Jin Lee ${ }^{1 \oplus}$, Soo Hyun $\mathrm{Kim}^{2 \oplus}$, Hee Jin Park ${ }^{2 \oplus}$, Sung Han Shim ${ }^{3 \oplus}$, Hee Yeon Jang ${ }^{3 \oplus}$, and Dong Hyun $\mathrm{Cha}^{2 * * \oplus}$ \\ ${ }^{1}$ Department of Obstetrics and Gynecology, CHA Ilsan Medical Center, CHA University, Goyang, Korea \\ ${ }^{2}$ Department of Obstetrics and Gynecology, CHA Gangnam Medical Center, CHA University, Seoul, Korea \\ ${ }^{3}$ Genetics Laboratory, Fertility Center of CHA Gangnam Medical Center, CHA University, Seoul, Korea
}

\begin{abstract}
Purpose: Trisomy 21, the cause of Down syndrome (DS) with various medical problems, is the most common aneuploidy during the fetal period. For diagnosis, a non-invasive screening test using maternal blood, which cannot be confirmed and invasive confirmation test with a risk of miscarriage, may be performed. The trophoblast retrieval and isolation of the cervix (TRIC) have been proposed by some researchers as an alternative to overcome the limitations of current tests. We experimented using TRIC to identify the possibility of trisomy 21 for the first time in Asia.

Materials and Methods: Three cases of DS were analyzed confirmed by invasive tests (chorionic villus sampling, amniocentesis). All samples of trophoblasts immediately were immersed in phosphate-buffered saline and processed with formalin for fixation. The trophoblasts were isolated using an anti-human leukocyte antigen-G antibody coupled to magnetic nanoparticles. $\beta$-human chorionic gonadotropin (hCG)-expressing cells were considered as trophoblast cells, and the detection rate calculated. DS was confirmed by fluorescence in situ hybridization (FISH).

Results: The mean trophoblast detection rate using $\beta$-hCG was $78.1 \%$, and the detection rate using FISH was $22.2 \%$. In all cases, the trisomy of chromosome 21 was identified.

Conclusion: Trophoblast can be obtained from the five weeks of gestation and has a high detection rate, so it is noted that it can replace the current prenatal genetic test. To realize the clinical application as a prenatal genetic test, we will need additional efforts to identify trisomy 21 as well as other chromosomal abnormalities in future large-scale studies.
\end{abstract}

Key words: Down syndrome, Fluorescent in situ hybridization, Noninvasive prenatal testing, Trophoblast, Trophblast retrieval and isolation from the cervix.

\section{Introduction}

According to the European Surveillance of Congenital Anomalies, trisomy 21, a common cause of Down syndrome (DS), accounts for more than half of all aneuploidy pregnancy [1]. About $50 \%$ of neonates born with DS have cardiac defects (ventricular septal defects and endocardial cushion defects), and 12\% have gastrointestinal abnormalities (esophageal atresia, Hirschsprung disease, and duodenal atresia). Children have health problems (hearing loss, severe optical refractive errors, cataracts, obstructive sleep apnea, thyroid disease, and leukemia) [2]. In adults, one-third of women deliver DS babies, and almost men are

Received: 24 May 2020, Revised: 29 July 2020, Accepted: 8 August 2020, Published: 31 December 2020

*Corresponding author: Dong Hyun Cha, Ph.D. (DD https://orcid.org/0000-0003-0722-1714

Department of Obstetrics and Gynecology, CHA Gangnam Medical Center, CHA University, 566 Nonhyeon-ro, Gangnam-gu, Seoul 06135, Korea.

Tel: +82-2-3468-3132, Fax: +82-2-3468-3039, E-mail: chadh001@ chamc.co.kr

Conflict of interest: The authors declare that they do not have any conflicts of interest.

(C) This is an open-access article distributed under the terms of the Creative Commons Attribution Non-Commercial License (http://creativecommons.org/licenses/by-nc/4.0/) which permits unrestricted non-commercial use, distribution, and reproduction in any medium, provided the original work is properly cited.

(c) Copyright 2020 by the Korean Society of Medical Genetics and Genomics 
infertile [3]. DS can affect not only the economic problems of medical costs but also social adaptation, as it is accompanied by varying degrees of mental retardation [4]. Therefore, prenatal testing for DS provides essential information to pregnant women preparing for childbirth.

Currently, prenatal tests that can identify DS are divided into diagnosis and screening. Diagnosis is a confirmative test to determine whether a fetus has chromosomal abnormalities. Confirmative tests such as chorionic villous sampling (CVS) and amniocentesis are invasive and performed to analyze chromosomes in cells derived from fetus extracted from the chorion and amniotic fluid. Although the detection rate for DS is more than 99\%, it is reported that the procedure-related pregnancy loss rated is 1-2\%, which makes maternal anxiety [5]. On the other hand, the screening method predicts the risk of fetus' chromosomal abnormalities and analyzes fetal DNA in the blood serum of pregnant women. The detection rate of DS using a non-invasive prenatal test (NIPT) is known to be 98-99\% [6]. If a high-risk result is found for chromosomal abnormality in NIPT, the invasive test should be done. People hope for an innovative technology that overcomes the limitations (invasiveness, need to confirm) of the current testings, so some researchers have suggested the alternative testing using trophoblast retrieval and isolation of the cervix (TRIC).

The TRIC is based on the theory that during the 5-12 weeks of gestation, a portion of the extravillous trophoblast may fall from the edge of the placenta, enter the uterine cavity, then reach to the cervix and be isolated. Many studies have shown that samples taken in the same way as the Papanicolaou (Pap) test, performed in early pregnancy, have reported a detection rate of 75-100\% of the TRIC. TRIC has confirmed the higher detection rate of trophoblasts than cell-free fetal DNA (cff DNA) using maternal blood. Various studies are currently underway to detect chromosomal abnormalities using TRIC. Our team has already reported on how to separate more efficiently TRIC cells using post-fixation [7]. This study aimed to confirm the diagnosis of DS, the most common aneuploidy in the prenatal period, using our post-fixation TRIC method for the first time in Asia.

\section{Materials and Methods}

\section{Patient selection}

We received the IRB approval of the Gangnam Cha Medical Center (GCl-17-38). The study conducted with the informed consent of all participants between August and November 2018. The clinician who took a sample knew that the fetus had a DS, but the researcher who processed the tissue and conducted it did not know about it. All participants were singleton pregnant women within 17 weeks of gestation. Three cases of DS were analyzed by invasive confirmatory tests (CVS, amniocentesis) due to increased nuchal translucency, positive maternal biochemical serum marker, advanced maternal age.

\section{Endocervical sampling and fixation}

The samples are taken in the same way as the Pap test using the cytobrush. To prevent cell aggregation caused by alcohol, we immediately immersed in PBS and transferred to the laboratory. The mucus of samples was removed using 3\% acetic acid (300 $\mu \mathrm{L} / 10 \mathrm{~mL}$ ) at room temperature for 5 minutes and then washed and treated with trypsin-EDTA. The washed cells were then fixed with $3.7 \%$ formalin at $4^{\circ} \mathrm{C}$ for 10 minutes. Fixed cells centrifuged at $900 \mathrm{~g}$ for 5 minutes stored at $4^{\circ} \mathrm{C}$ immediately after washing.

\section{Isolation of trophoblast cells}

The $20 \mu \mathrm{L}$ of $250 \mathrm{~nm}$ magnetic nanoparticles conjugated to goat anti-mouse IgG antibody (Clemente Associate, Madison, CT, USA) was incubated with Mouse anti-human leukocyte antigen (HLA)-G antibody ( $5 \mu \mathrm{g}$, Clone 4H84, BD Biosciences, Pharminge, $C A, U S A)$ for overnight at $4^{\circ} \mathrm{C}$. The fixed cells conjugated $\mathrm{HLA}-\mathrm{G}$ coupled nanoparticles and incubated overnight at $4^{\circ} \mathrm{C}$ with mixing. Using magnetic immobilization, the bounding cells, which were considered trophoblast cells, were collected and washed with cold PBS.

\section{Immmunochemistry}

Since trophoblast secrets human chorionic gonadotropin (hCG) specifically, the experimental method for detecting trophoblast using it is as follows.

The HLA-G positive cells suspended in $200 \mu \mathrm{L}$ of PBS were centrifuged onto a slide using a Cytospin 7620 (Wescor Inc., Logan, UT, USA) at 1,500 rpm for 5 minutes. The cells were dried on slides and blocked for 1 hour or more in PBS with 3\% BSA at $4^{\circ} \mathrm{C}$. The slides were labeled with a $\beta$-hCG primary antibody (1:50 dilution) overnight at $4^{\circ} \mathrm{C}$. The slides were incubated with the primary antibody at $4^{\circ} \mathrm{C}$ overnight. Then the slide was incubated with Alexa Fluor ${ }^{\circledR} 555$ goat anti-mouse lgG (Invitrogen, Carlsbad, CA, USA) at $4^{\circ} \mathrm{C}$ for 1 hour. Then, the cells dyed at room temperature with 4', 6-diamidino-2-phenylindole dihydrochloride for 10 minutes. The cells were observed under ZEISS Axio Imager 2 fluorescence microscope (Carl Zeiss, Thornwood, NY, USA). 


\section{Fluorescence in situ hybridization}

According to the manufacturer's protocol, trisomy 21 was confirmed using FISH probes for chromosome 21 using TelVysion 21q SpectrumOrage (Abbott Molecular, Des Plaines, IL, USA) and signals were analyzed using Zeiss AXIO Imager A2 fluorescent microscope (Carl Zeiss) with the Isis FISH imaging system.

\section{Results}

Of the three cases, advanced maternal age was 2 cases, and multiparous was 1 case (Table 1). The average number of total endocervical cells was 4.7×105, and $\mathrm{HLA}-\mathrm{G}(+$ ) was 1,440 (Table 2). The mean trophoblast detection rate using $\beta$-hCG was $78.1 \%$, and the detection rate using FISH was $22.2 \%$, which was

Table 1. Characteristics 3 cases of DS diagnosed for TRIC analysis

\begin{tabular}{ccccccc}
\hline Sample number & Age & Gravity & Parity & GA & Confirm test & Indication \\
\hline 1 & 38 & 3 & 2 & $13+5$ & CVS & Increased NT \\
2 & 41 & 1 & 0 & $16+0$ & Amniocentesis & Integrated test-DS, NTD high risk \\
3 & 34 & 1 & 0 & $16+5$ & Amniocentesis & Integrated test-DS, NTD high risk \\
\hline
\end{tabular}

DS, down syndrome; TRIC, trophoblast retrieval and isolation of the cervix; GA, gestational age (weeks); CVS, chorionic villous sampling; NT, nuchal translucency; NTD, neural tube defect.

Table 2. Cytologic characteristics of 3 cases using TRIC

\begin{tabular}{ccccc}
\hline \multirow{2}{*}{ Sample number } & \multirow{2}{*}{ Total endocervical cells } & HLA-G positive cells & \multicolumn{2}{c}{ Detection rate (\%) } \\
\cline { 4 - 5 } & $5.3 \times 10^{5}$ & 900 & 81.5 & FISH \\
\hline 2 & $4.2 \times 10^{5}$ & 1,670 & 65.7 & 24.0 \\
3 & $4.5 \times 10^{5}$ & 1,750 & 78.0 & 23.3 \\
Mean & $4.7 \times 10^{5}$ & 1,440 & 78.1 & 19.4 \\
\hline
\end{tabular}

TRIC, trophoblast retrieval and isolation of the cervix, HLA, human leukocyte antigen; $h C G$, human chorionic gonadotropin; FISH, fluorescent in situ hybridization.
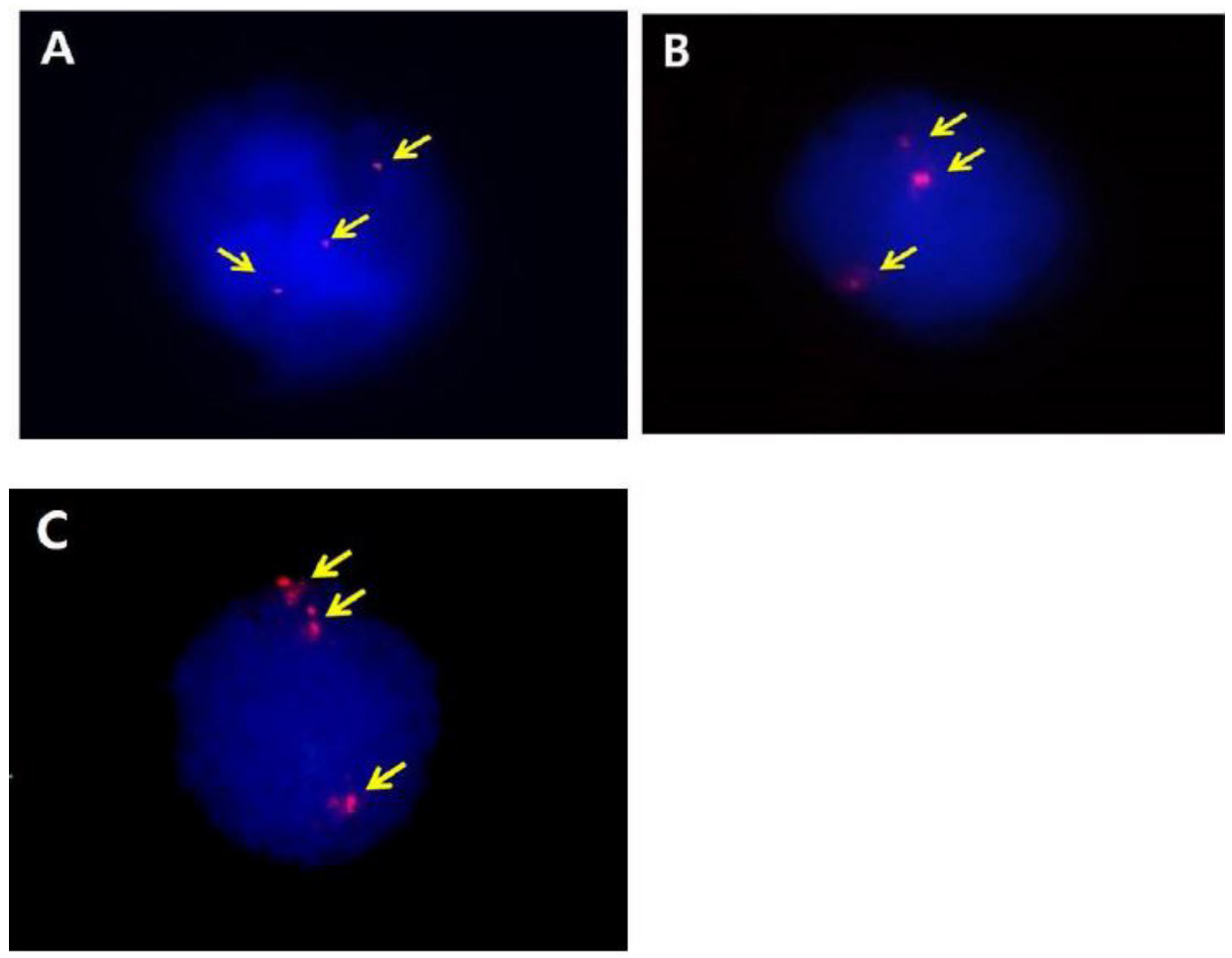

Fig. 1. Fluorescence in situ hybridization result for Down syndrome diagnostic analyzed with trophoblast retrieval and isolation of the cervix. The yellow arrow points to chromosome 21. (A-C) correspond to samples number 1-3 cases, respectively. 
3.4 times lower (Table 2). DS identified chromosome 21 using FISH (Fig. 1).

\section{Discussion}

DS is affected by the maternal age because it is known to be caused by nondisjunction during meiosis. While women's socioeconomic status gradually improves, the incidence of DS pregnancy according to advanced maternal age has increased by about 33\% during four decades [8,9]. Therefore, the importance of prenatal genetic testing is emphasized to prepare for the birth of DS.

Pregnant women hope to be confirmed with high accuracy tests for genetic abnormalities without threatening their fetus' well-being. To meet these needs, the cff DNA analysis using maternal blood, presented by Lo et al. [10] in 1997, led to the rapid growth of the NIPT market. Compared to conventional screening tests, the NIPT market has increased dramatically because of the higher sensitivity and specificity and lower false positive rate of aneuploidy for 13,18,21, and sex chromosomes, as well as the absence of miscarriage risk. NIPT utilizes a fetal DNA fraction derived from apoptotic trophoblast cells programmed cell death. The traditional NIPT fragment is $160 \mathrm{bp}$ and is generally known to be about 10\%. For this reason, cffDNA derived from maternal and placental is affected by placental mosaicism, vanishing twin, occult maternal malignancy [11]. A low fraction of less than 4\% causes assay failure, high assay variance, and is influenced by the maternal weight or early gestational age [12]. NIPT can't overcome the limitations of the screening test. Ultimately, there is no non-invasive diagnostic test yet.

In 1971, Shettles [13] first confirmed the presence of trophoblast from the cervix. For half a century, various studies have proven that the trophoblast obtained from the cervix is intact fetal DNA [14]. It has been reported that this makes it possible to identify fetal DNA regardless of the maternal weigh [15]. On average, a woman notices pregnancy at 5.5 weeks. When pregnancy is confirmed, the prenatal test, including a screening test for cervical cancer, is performed at the early pregnancy [16]. The safety of the cytobrush used at this time has already been proven [17]. Most TRIC studies use cytobrush for trophoblast sampling, which is the same as the Pap test. Also, it has been reported that trophoblast can be obtained from the five weeks of gestation $[18,19]$. This means that pregnant women do not have to spend extra time or perform any additional procedures, and can check DS at the earliest of the existing test. It can also provide mothers sufficient information and time to plan for their future.

Prenatal genetic testing using TRIC is the hope of non-invasive confirmatory testing that satisfies all conditions of early testing, conciseness, safety, and intact fetal DNA. The key to the clinical application of TRIC depends on how purely the trophoblast is isolated. Our research team defined the basic concept of TRIC in four-steps (sampling, fixation, isolation of trophoblast, analysis), and among them, reported a study on how to increase the efficiency of cell isolation in the cell fixation step (called as post-fixation method) [7]. Using the post-fixation method, the possibility of a diagnosis of DS, which is the most common chromosome abnormality in the prenatal period, was confirmed in the study. However, unlike the FISH results that can confirm the pure trophoblast, the rate of trophoblast detection in immunochemistry is nearly four times different. This suggests that non-trophoblast cells have not been sufficiently isolated by TRIC using immunochemistry. This remains a challenge for us to improve on the TRIC.

This study has the strength of being the first study to confirm the DS diagnosis in Asia by TRIC. On the other hand, the limitations are that the small sample size and the number of gestational age are extensive. Our research expected to confirm the diagnosis of other aneuploidies using TRIC in the future, and to be used as a basis for confirmatory tests that can replace NIPT and conventional invasive tests by combining NGS technology.

\section{Acknowledgements}

This journal was awarded the Best Speech Award at the 2019 fall conference of the Korean Society of Medical Genetics and Genomics.

\section{References}

1. Wellesley D, Dolk H, Boyd PA, Greenlees R, Haeusler M, Nelen $V_{\text {, et al. }}$ Rare chromosome abnormalities, prevalence and prenatal diagnosis rates from population-based congenital anomaly registers in Europe. Eur J Hum Genet 2012;20:521-6.

2. Bull MJ; Committee on Genetics. Health supervision for children with Down syndrome. Pediatrics 2011;128:393-406.

3. Scharrer S, Stengel-Rutkowski S, Rodewald-Rudescu A, Erdlen E, Zang KD. Reproduction in a female patient with Down's syndrome. Case report of a $46, X Y$ child showing slight phenotypical anomalies, born to a 47, XX, 21 mother. Humangenetik 1975;26:207-14.

4. Antonarakis SE, Skotko BG, Rafii MS, Strydom A, Pape SE, Bianchi DW, et al. Down syndrome. Nat Rev Dis Primers 2020;6:9. 
5. American College of Obstetricians and Gynecologists' Committee on Practice Bulletins-Obstetrics; Committee on Genetics; Society for Maternal-Fetal Medicine. Practice bulletin no. 162: prenatal diagnostic testing for genetic disorders. Obstet Gynecol 2016;127:e108-22.

6. Dashe JS. Aneuploidy screening in pregnancy. Obstet Gynecol 2016;128:181-94.

7. Lee MJ, Kim SH, Shim SH, Jang HY, Park HJ, Cha DH. Optimization protocol of fixation method for trophoblast retrieval from the cervix (TRIC): a preliminary study. Diagnostics (Basel) 2020;10:300.

8. Loane M, Morris JK, Addor MC, Arriola L, Budd J, Doray B, et al. Twenty-year trends in the prevalence of Down syndrome and other trisomies in Europe: impact of maternal age and prenatal screening. Eur J Hum Genet 2013;21:27-33.

9. Shin M, Besser LM, Kucik JE, Lu C, Siffel C, Correa A; Congenital Anomaly Multistate Prevalence and Survival Collaborative. Prevalence of Down syndrome among children and adolescents in 10 regions of the United States. Pediatrics 2009;124:1565-71.

10. Lo YM, Corbetta $N$, Chamberlain PF, Rai V, Sargent IL, Redman CW, et al. Presence of fetal DNA in maternal plasma and serum. Lancet 1997;350:485-7.

11. Committee opinion no. 640: cell-free DNA screening for fetal aneuploidy. Obstet Gynecol 2015;126:e31-7.

12. Ashoor G, Syngelaki A, Poon LC, Rezende JC, Nicolaides KH. Fetal fraction in maternal plasma cell-free DNA at 11-13 weeks' gestation: relation to maternal and fetal characteristics. Ultrasound Obstet Gy- necol 2013;41:26-32.

13. Shettles LB. Use of the $Y$ chromosome in prenatal sex determination. Nature 1971;230:52-3.

14. Moser G, Drewlo S, Huppertz B, Armant DR. Trophoblast retrieval and isolation from the cervix: origins of cervical trophoblasts and their potential value for risk assessment of ongoing pregnancies. Hum Reprod Update 2018;24:484-96.

15. Fritz $R$, Kohan-Ghadr HR, Sacher $A$, Bolnick AD, Kilburn BA, Bolnick $J M$, et al. Trophoblast retrieval and isolation from the cervix (TRIC) is unaffected by early gestational age or maternal obesity. Prenat Diagn 2015;35:1218-22.

16. Branum AM, Ahrens KA. Trends in timing of pregnancy awareness among US women. Matern Child Health J 2017;21:715-26.

17. Orr JW Jr, Barrett JM, Orr PF, Holloway RW, Holimon JL. The efficacy and safety of the cytobrush during pregnancy. Gynecol Oncol 1992;44:260-2.

18. Kadam L, Jain C, Kohan-Ghadr HR, Krawetz SA, Drewlo S, Armant DR. Endocervical trophoblast for interrogating the fetal genome and assessing pregnancy health at five weeks. Eur J Med Genet 2019;62:103690.

19. Jain CV, Kadam L, van Dijk M, Kohan-Ghadr HR, Kilburn BA, Hartman $C_{\text {, et }}$ al. Fetal genome profiling at 5 weeks of gestation after noninvasive isolation of trophoblast cells from the endocervical canal. Sci Transl Med 2016;8:363re4. 